\title{
Design of Elliptic Reflective LED Surgical Shadowless Lamps Using Mathematical Optical Tracing Algorithms
}

\author{
Cheng-Tang Pan, ${ }^{1}$ Yi-Chian Chen, ${ }^{1}$ Tsung-Lin Yang, ${ }^{1}$ \\ Po-Hsun Lin, ${ }^{1}$ Po-Hung Lin, ${ }^{1}$ and C. C. Hsieh ${ }^{2}$ \\ ${ }^{1}$ Department of Mechanical and Electro-Mechanical Engineering, Center for Nanoscience and Nanotechnology, \\ National Sun Yat-Sen University, Kaohsiung 80424, Taiwan \\ ${ }^{2}$ Department of the Mechanical and Automation Engineering, National Kaohsiung First University of Science and Technology, \\ Kaohsiung 811, Taiwan
}

Correspondence should be addressed to C. C. Hsieh; cchsieh@nkfust.edu.tw

Received 12 June 2014; Accepted 11 September 2014; Published 29 September 2014

Academic Editor: Teen-Hang Meen

Copyright (C) 2014 Cheng-Tang Pan et al. This is an open access article distributed under the Creative Commons Attribution License, which permits unrestricted use, distribution, and reproduction in any medium, provided the original work is properly cited.

Traditional surgical shadowless halogen lamps are generally designed as projection type with many light bulbs, which can produce not only mercury pollution but also heat radiation that are serious problems to patient. The study utilized Runge-Kutta methods and mathematical algorithms to design and optimize the freeform lens. The LED (light-emitting diode) was adopted to replace the traditional halogen lamp. A uniform lens was designed and fabricated based on the energy conservation. At first, the light field of LED is concentrated through the freeform lens to improve the optical efficiency. Second, the three-shell elliptic curves are applied to the reflective surgical shadowless lamps, where only few LED chips are needed. Light rays emitting from different directions to the target plane can achieve the goal of shadowless. In this study, the LED's luminance flux is $1,895 \mathrm{~lm}$. The shadow dilution on the target plane is $54 \%$. $E_{c}$ (central illuminance) is $114,900 \mathrm{lux}$, and the $d_{50} / d_{10}$ is $57 \%$ which is higher than the regulation by $7 \%$, whereas the power consumption is only $20 \mathrm{~W}$. The energy of reflective surgical shadowless lamps can save more than $50 \%$, compared with the traditional projective one.

\section{Introduction}

1.1. Background. The light source of traditional surgical shadowless lamps is usually halogen lamp, but it can cause serious environmental pollution. In order to achieve the goal of environmental protection, light emitting diode (LED) is applied to this research as light source; the advantages of LED are cold light, low color temperature, high color rendering, and long life term $[1,2]$, that solved the heat radiation problems and the color performance of the surgical parts can be enhanced.

Surgical shadowless lamp which is designed to reduce the shadow generated by hands or surgical tools to meet the regulation plays a critically important role in the surgery room. But the energy consumption of projective shadowless lamps is too high to generate the problem of irradiance heat because there were many halogen lamps used as light source. Compared with projective surgical lamps, reflective shadowless lamps have been developed for a long time, a surgical lamp designed by an elliptic curve reflector was invented by Dorman in 1970 [3], and the authors brought up another elliptic curve assembled by many long and thin lenses in 1979 [4]. Michael and Holt brought up a cone surface reflector combined with a refractive lens below the reflector [5]. Ries and Muschaweck developed three-dimensional design methods known as tailored freeform method, which can calculate asymmetric or symmetric optics surface [6]; Fournier proposed a design methods that can ease the design of freeform reflectors and provide efficient, cost-effective solutions that avoid unnecessary energy consumption and light pollution in 2010 [7]. Canavesi et al. proposed a fast and simple algorithm to design two-dimensional reflector surfaces in 2012. Compared with the linear programming method, the direct calculation algorithm can produce 


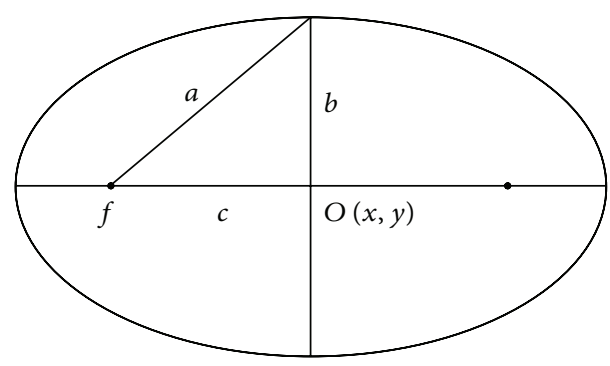

FIGURE 1: Ellipse diagram.

the same result in shorter time [8]. In 2012, Chen et al. proposed a freeform reflector design method of uniform illumination. Moreover, the equation and the boundary condition could be determined by the characteristics of light source [9].

1.2. Motivation. Illumination system can be separated into three different parts, projection, reflection, and optical fiber. Freeform lenses are wildly used in projection system; light can be concentrated/diffused while going through two different materials. We can determine any kind of light field with freeform lens by using calculation and simulation process. The light in reflection system can be reflected to target plane by several reflective layers, which is calculated through math method. The illumination system in surgical room is required to have high illumination, high color rendering, glare free, and little irradiance.

\section{Design Methods}

2.1. Stacked Elliptic Curve. The first part is stacked elliptic curve reflector. There are several methods to design and fabricate surgical lamps, such as refracting light to target plane by Fresnel lens which is composed of many different prisms [10] and utilizing continuous profiles to enhance the uniformity of light distribution [11]. Moreover, multireflector surgical lamp which combined parabola surface and elliptic curve as reflector and placed the light source at cofocus was brought up in 1990 by Harry [12].

The reflector is stacked by three elliptic curves with different focuses. Each elliptic curve is an independent light source that makes light emit to the target plane from different angles, and the goal of the shadowless can be achieved. Equation (1) and Figure 1 are ellipse equation and ellipse diagram which are applied to the calculation of the stacked elliptic curves:

$$
\frac{\left(x-x_{1}\right)^{2}}{a^{2}}+\frac{\left(y-y_{1}\right)^{2}}{b^{2}}=1,
$$

where $a$ and $b$ are half-length of major axis and minor axis, respectively. The $x_{1}, y_{1}$ are the coordinates of the center point of the ellipse.

The depth of reflector and the position of light source which is one of the focuses of the first elliptic curve have to be determined at the beginning. The diameter of light field

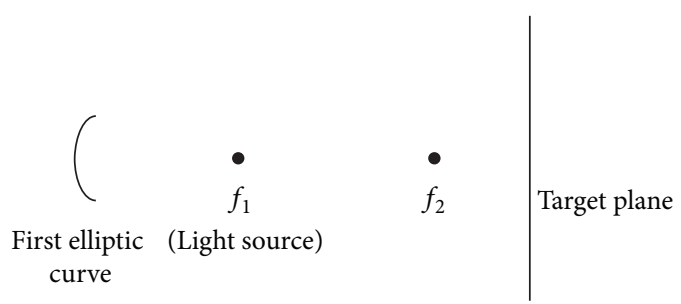

FIgURE 2: First elliptic curve and light source.

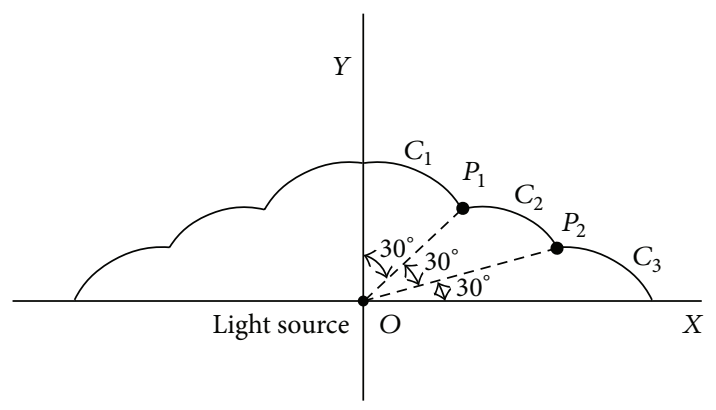

FIGURE 3: Predicted curve of stacked ellipse.

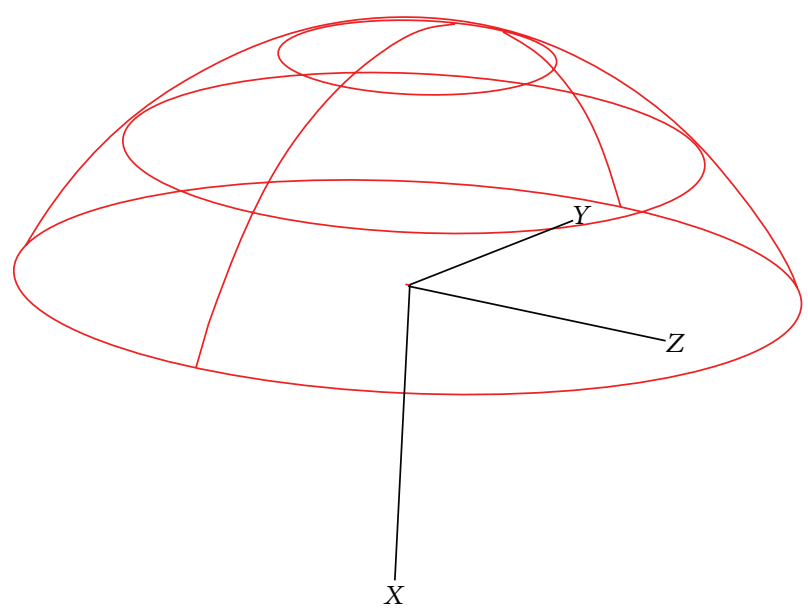

FIGURE 4: The structure of stacked elliptic curve.

on the target plane is set as $30 \mathrm{~cm}$. Figure 2 shows that the target plane should be set at $1 \mathrm{~m}$ below the emission plane and $f_{2}$ is the second focus of ellipse and the light passing through $f_{2}$ can form a circle pattern with $30 \mathrm{~cm}$ diameter on the target plane according to the regulation [13]. The whole elliptic curve is stacked by 3 curves, and each of them has $30^{\circ}$ included angle as shown in Figure 3, where $P_{1}$ and $P_{2}$ are the intersection points between $C_{1}, C_{2}$, and $C_{3}$. After $C_{1}$ is sketched, the coordinate of $P_{2}$ can be obtained by using Pro/E [14] engineering software. The $f_{2}$ of $C_{2}$ is determined by the diameter of light field, and the center point can be calculated as well. Then, the second elliptic curve can be sketched. The whole elliptic curve can be obtained by repeating the processes above, as shown in Figure 4. 


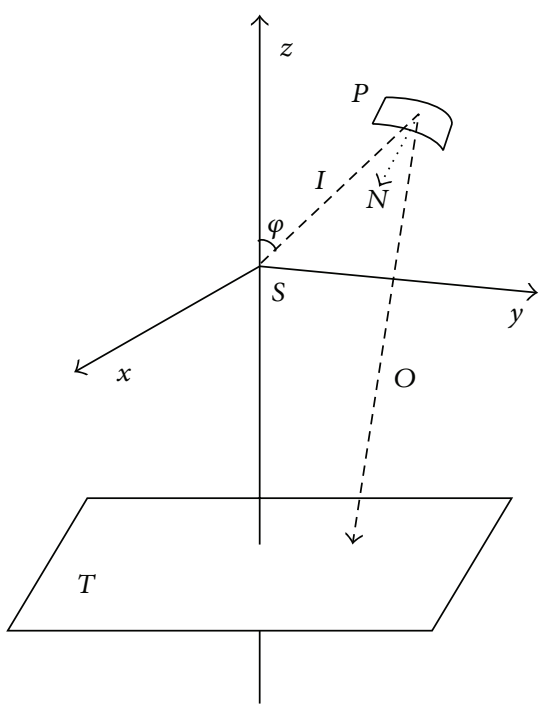

Figure 5: Sketch of reflection.

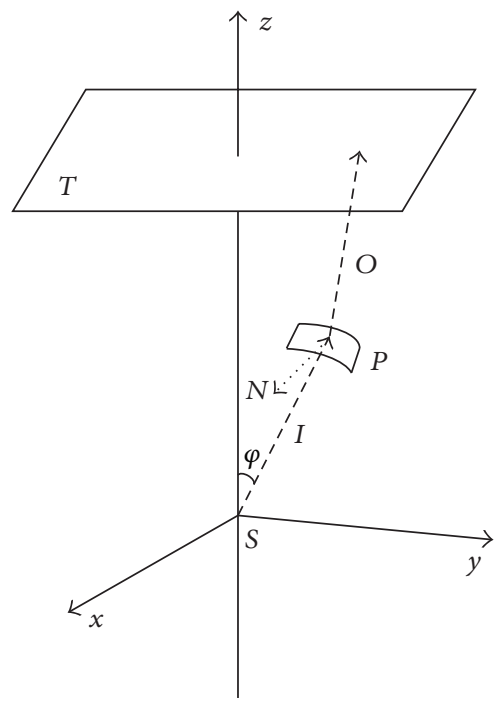

FIGURE 6: Sketch of refraction.

2.2. Uniform Free-Form Lens. LED light source can be considered a Lambertian distribution, and the light emission of half intensity angle is $60^{\circ}$ that affects the uniformity of light distribution on the target plane. A secondary optical uniform lens is fabricated to solve the problems and the uniformity can be enhanced by using this design $[15,16]$. The light distribution of a bare LED is Lambertian distribution, whose light distribution is defined as cosine exponential function of angle $\theta$. The light intensity function is expressed in (2). The light intensity as a function of angle $\theta$ from central axis is $I_{S}(\theta)=I_{O} \cos ^{m}(\theta)$, where $I_{O}$ is a constant intensity, $I_{S}$ is light source, and $m$ value is determined by the half intensity angle $\theta_{0.5}$ of LED. The half intensity angle of the LED is 60 degree;

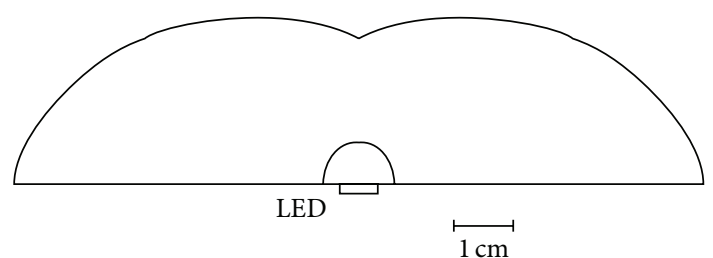

FIGURE 7: Profile of the free-form lens.

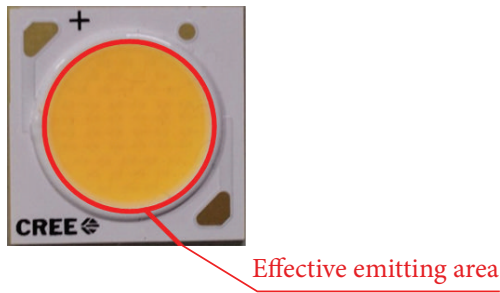

$10 \mathrm{~mm}$

Figure 8: The CREE CXA1816 LED chip.

then the $m$ value is 1 . The light field function of light source can be expressed as (3)

$$
\begin{aligned}
I(\theta) & =I_{O} \cos ^{m}(\theta), \\
m & =\frac{-\ln 2}{\ln \left(\cos \theta_{0.5}\right)}, \\
I_{S}(\theta) & =I_{O} \cos (\theta) .
\end{aligned}
$$

At first, the Cartesian plane and a spherical coordinate are established with the same origin of coordinate, where $\theta$ is the angle between the $x$-axis and projection of a vector on the $x$ $y$ plane, and $\varphi$ is the angle between the $z$-axis and a vector. The coordinate in the Cartesian plane is set as $(x, y, z)$. The equation of curve can be expressed as in Figures 5 and 6 and (4):

$$
\begin{gathered}
x=x(\theta, \varphi), \\
y=y(\theta, \varphi), \\
z=z(\theta, \varphi), \\
(\theta, \varphi) \in D .
\end{gathered}
$$

The vector equation of curve can be expressed as

$$
p=p(\theta, \varphi)=(x(\theta, \varphi), y(\theta, \varphi), z(\theta, \varphi)),
$$

where $\rho(\theta, \varphi)$ is the length of vector $p$, and $x, y, z$ are shown in $(6)$,

$$
\begin{aligned}
& x(\theta, \varphi)=\rho \times \sin \varphi * \cos \theta, \\
& y(\theta, \varphi)=\rho \times \sin \varphi * \sin \theta, \\
& z(\theta, \varphi)=\rho \times \cos \varphi .
\end{aligned}
$$




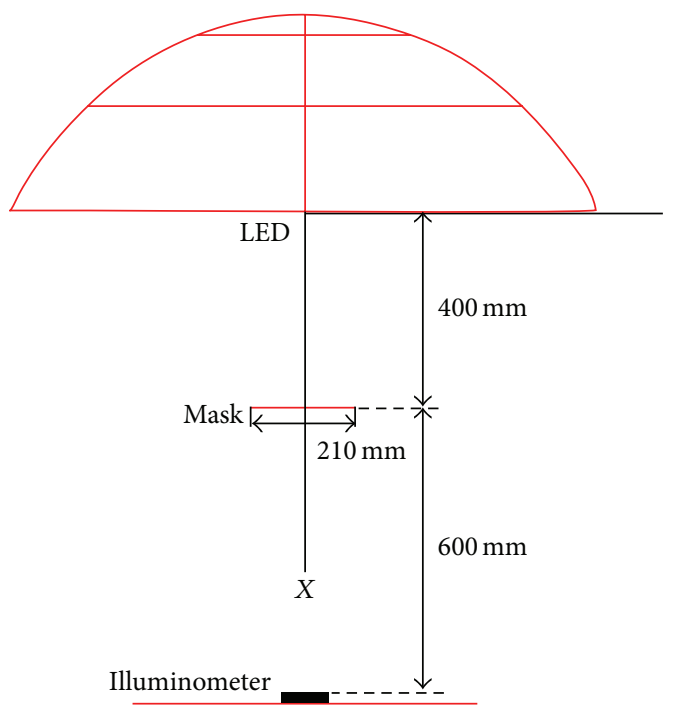

(a)

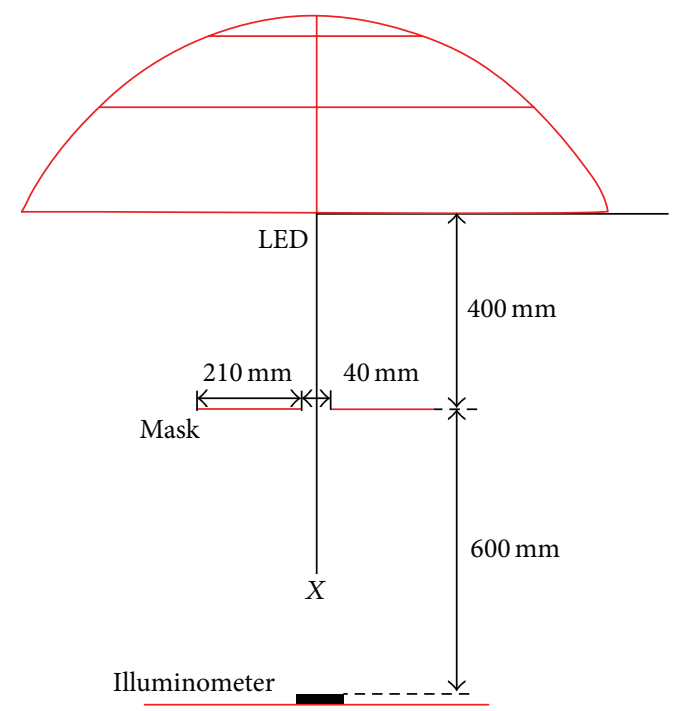

(b)

FIgURE 9: (a) Single mask and (b) double masks.
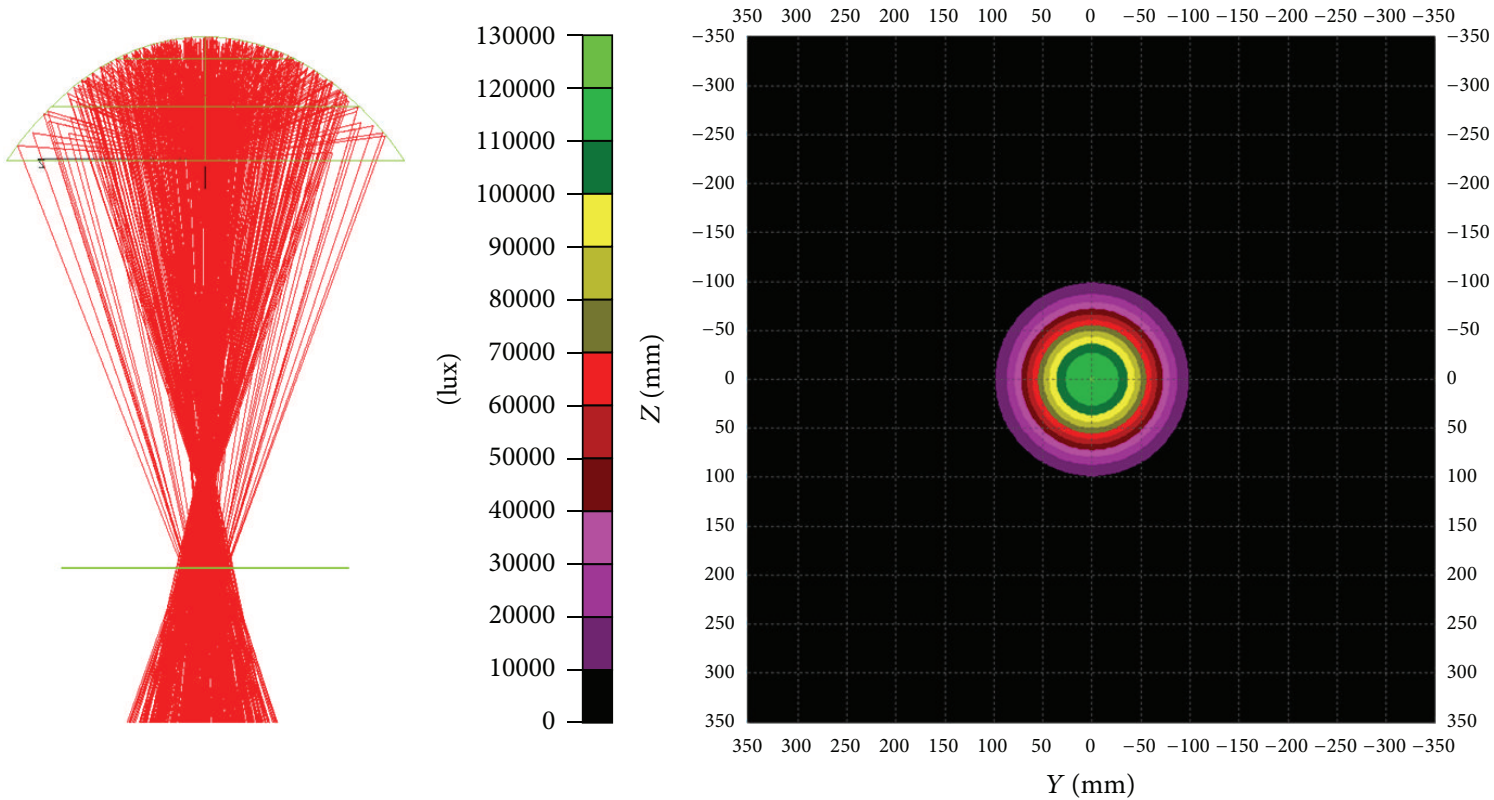

Figure 10: Simulation result of the light emission and irradiance map without mask.

The direction vector of incident light $\mathbf{I}$ is set as the unit vector from light source to uniform lens, as shown in (7),

$$
\begin{aligned}
& I_{x}=\sin \varphi \times \cos \theta, \\
& I_{y}=\sin \varphi \times \sin \theta, \\
& I_{z}=\cos \varphi .
\end{aligned}
$$

And $\mathbf{O}$, the direction vector of emergent light, is the unit vector of emergent light that directs to the target plane $\mathbf{T}$ after refracting/reflecting from the lens curve $\mathbf{P}$. Setting coordinate of lens curve $\mathbf{P}$ as $\left(p_{x}, p_{y}, p_{z}\right)$, the coordinates of target plane $\mathbf{T}$ are $(x, y, z)$. Therefore, $\mathbf{O}$ can be expressed as

$$
\begin{aligned}
& O_{x}=\frac{\left(x-p_{x}\right)}{|t-p|}, \\
& O_{y}=\frac{\left(y-p_{y}\right)}{|t-p|}, \\
& O_{z}=\frac{\left(z-p_{z}\right)}{|t-p|}
\end{aligned}
$$



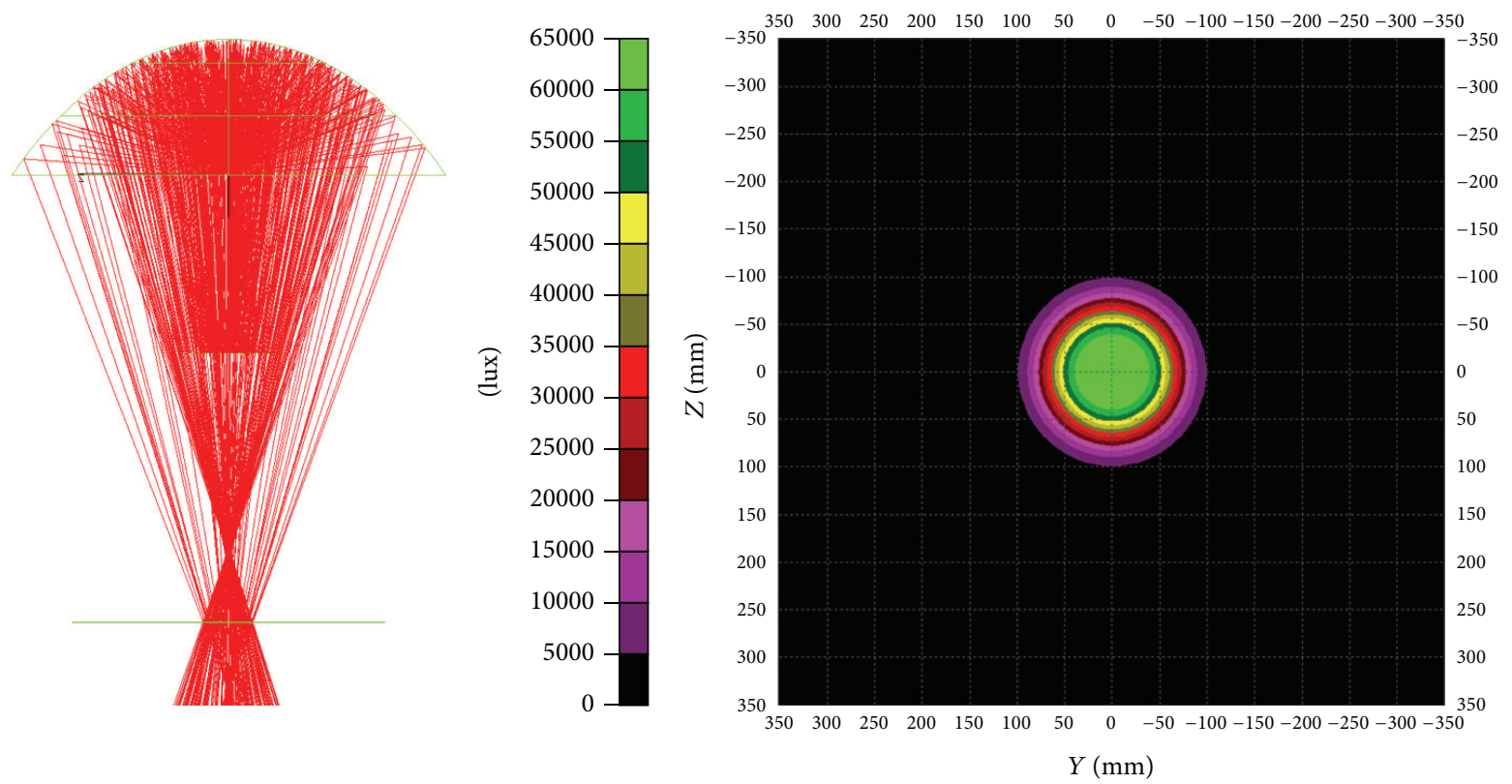

FIGURE 11: Simulation result of the light emission and irradiance map with single mask.
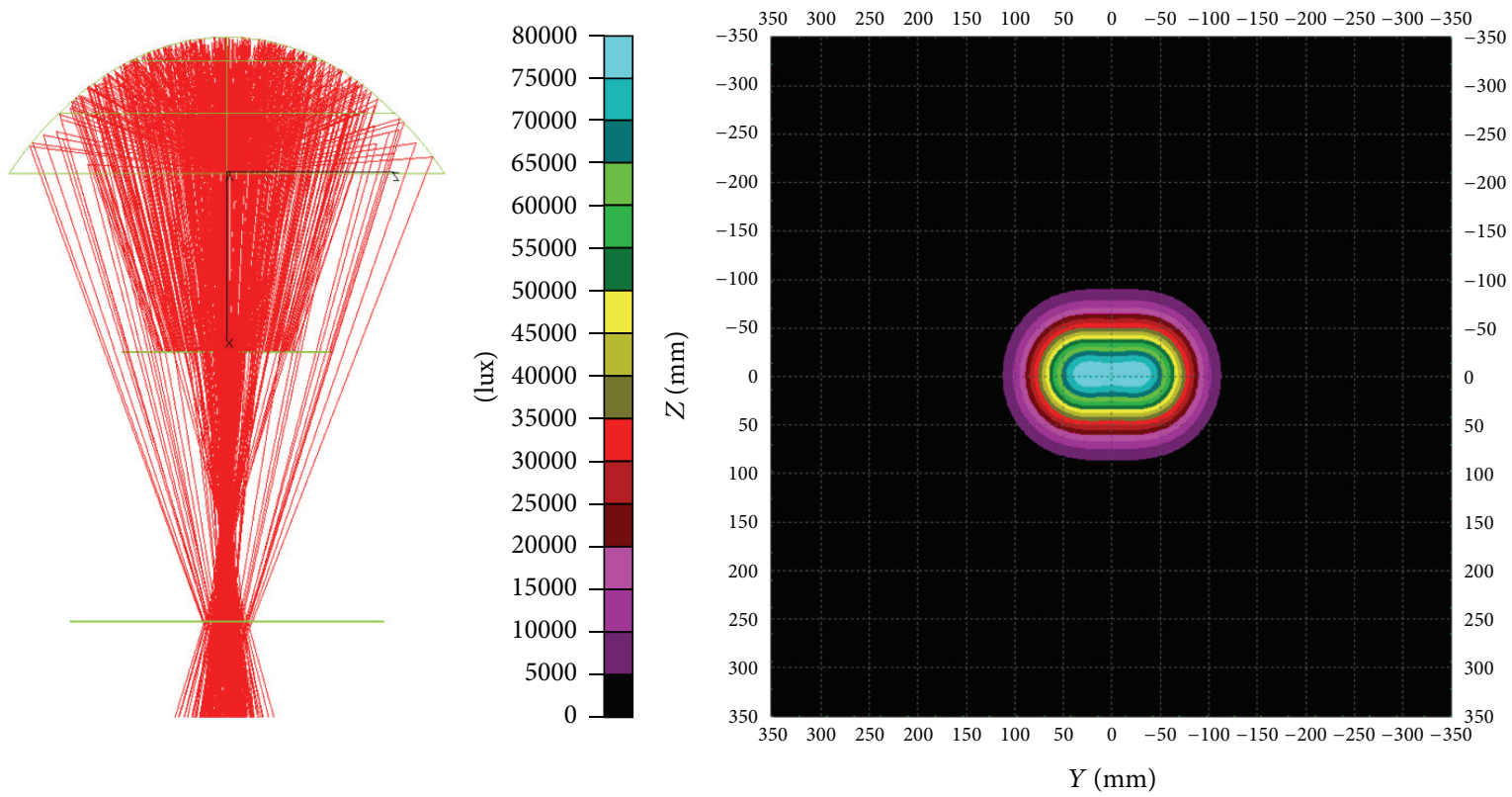

FIGURE 12: Simulation result of the light emission and irradiance map with double mask.

After substituting (6) into Snell's law, lens curve $\mathbf{P}$ can be obtained as

$$
\begin{aligned}
& x=\frac{N_{x}\left[n_{O}\left(z-p_{z}\right)-n_{I} I_{z}|t-p|\right]}{n_{O} N_{z}}+p_{x}+\frac{n_{I}}{n_{O}} I_{x}|\mathbf{t}-\mathbf{p}|, \\
& y=\frac{N_{y}\left[n_{O}\left(z-p_{z}\right)-n_{I} I_{z}|t-p|\right]}{n_{O} N_{z}}+p_{y}+\frac{n_{I}}{n_{O}} I_{y}|\mathbf{t}-\mathbf{p}| .
\end{aligned}
$$

Equation (10) shows the law of energy conservation:

$$
\int_{0}^{\theta} \int_{0}^{\varphi} \mathbf{I}(\theta, \varphi) \sin \varphi \mathrm{d} \varphi \mathrm{d} \theta=\int E(t) \mathrm{d} A,
$$

where $E$ is the total energy on the target plane. By solving the ordinary differential equation, $\mathbf{x}$ and $\mathbf{z}$ can be obtained. Pro/E is used to sketch the lens structures. Figure 7 is the cross-sectional figure of free-form lens sketched by Pro/E engineering software. 


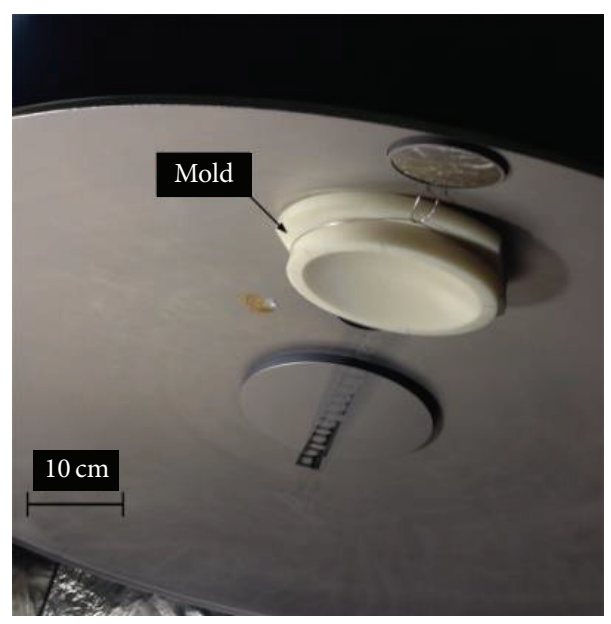

Figure 13: Vacuum evaporation process.
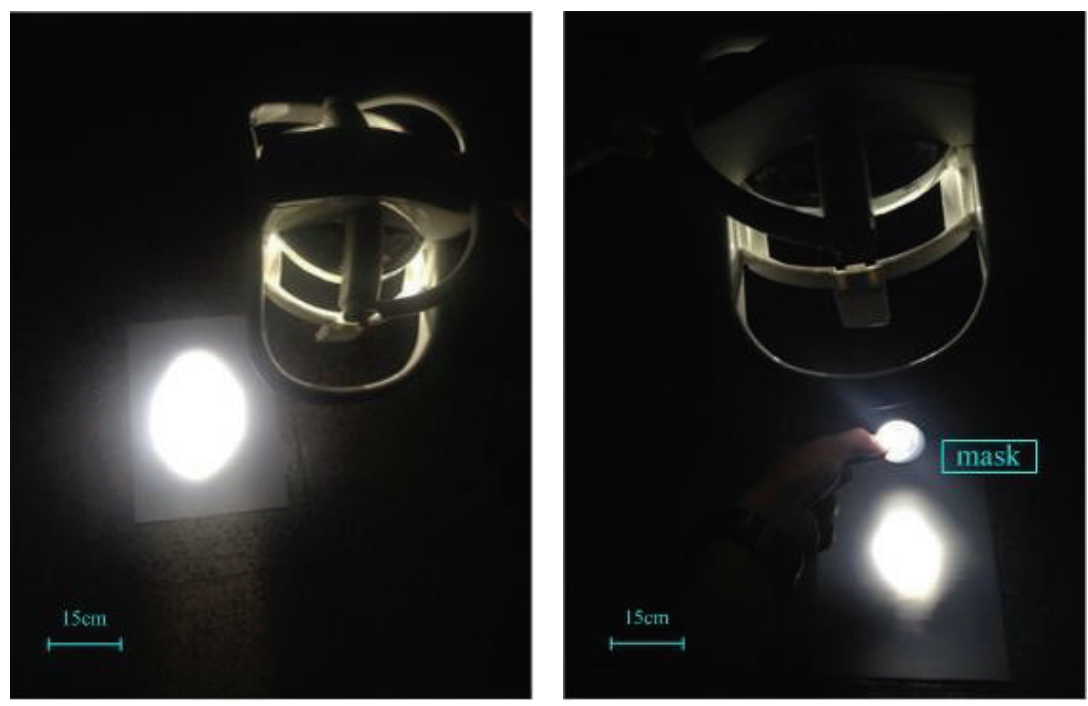

FIgURE 14: Actual emission pattern on target plane.

\section{Simulation of Stacked Shadowless Lamp}

3.1. Simulation. Table 1 shows that there was no significant advancement for $E_{c}$ and $d_{50} / d_{10}$ with more than three curves according to our simulation results. In addition, more curves could increase the difficulties of manufacture and cost. Summarizing the reasons above, we used 3 elliptic curves to compose the reflector.

The blocking of masks can lead to the decay of luminance. The central illuminance $\left(E_{c}\right)$ on the target plane which is $1 \mathrm{~m}$ below the light source should reach the minimum value of 40,000 lux and not exceed 160,000 lux according to the regulation. Table 2 shows the simulation result, and the $E_{c}$ with 1 or 2 masks meets the rule.

The light field diameter $d_{50} / d_{10}$ should be higher than 0.5 , where the $d_{50}$ illuminance reaches $50 \%$ of $E_{c}$ and the $d_{10}$ illuminance reaches $10 \%$ of $E_{c}$. The most important is that the shadow dilution should be higher than 0.5 when the beam is obstructed by one or two masks, with or without tubes [13].
TABLE 1: The simulation results of different curves.

\begin{tabular}{lcccc}
\hline Curve & 2 & 3 & 4 & 5 \\
\hline$E_{c}$ (lux) & 101,110 & 114,900 & 109,520 & 112,170 \\
$d_{50} / d_{10}$ & $57 \%$ & $64 \%$ & $63 \%$ & $63 \%$ \\
\hline
\end{tabular}

TABLE 2: Simulation results of the stacked shadowless lamp.

\begin{tabular}{lccc}
\hline Item & No mask & 1 mask & 2 masks \\
\hline$E_{c}(\operatorname{lux})$ & 114,900 & 64,983 & 78,032 \\
$d_{50} / d_{10}$ & 0.640 & 0.570 & 0.680 \\
Shadowless dilution & - & 0.553 & 0.645 \\
\hline
\end{tabular}

The commercial Trace Pro optical software is used to simulate the light emission of the stacked shadowless lamp. The LED we chose is fabricated by CREE incorporation, and the model number was CXA1816 with 1,895 lm. Figure 8 shows that the size of LED is $17.85 \times 17.82 \mathrm{~mm}^{2}$ and the effective emitting 
TABLE 3: Actual measurement data of stacked shadowless lamp.

\begin{tabular}{lccc}
\hline Item & No mask & 1 mask & 2 masks \\
\hline$E_{c}(\operatorname{lux})$ & 102,500 & 59,144 & 68,147 \\
$d_{50} / d_{10}$ & 0.680 & 0.540 & 0.559 \\
Shadowless dilution & - & 0.577 & 0.665 \\
\hline
\end{tabular}

area is $36 \pi \mathrm{mm}^{2}$. To ensure that the shadowless dilution does meet the regulation, the $E_{c}$ under one and two masks should be measured as shown in Figure 9, which is the structure diagram of simulation and the masks are placed $400 \mathrm{~mm}$ below the LED to block the light.

Figures 10,11 , and 12 are the simulation results and irradiance map of light emission with/without one or two masks, and the measurement results are listed in Table 2.

3.2. Construction and Measurement. 3D (three-dimensional) printing technology is used to fabricate the ABS resin mold of this stacked shadowless lamp. Vacuum evaporation process is adopted to coat the reflective aluminum-based material on the surface of the mold, as shown in Figure 13.

Figure 14 shows the real emission pattern on the target plane. From the photo, there was no shadow on the target plane while a mask is placed between light source and target plane. The actual measurement data is listed in Table 3, which is measured by illuminometer.

\section{Conclusion}

In this research, we brought up a modified stacked elliptic curves shadowless lamp combined with uniform free-form lens. LED is used to the light source. The mold is fabricated by $3 \mathrm{D}$ printing technology and vacuum evaporation process. The $E_{c}$ on the target plane reaches 102,500 lux and $d_{50} / d_{10}$ is higher than regulation one by $7 \%$. The shadowless dilution is about $57 \%$, which is higher than regulation one by $7 \%$. Through this design, we can achieve the goals of long life term and environmental protection. In addition, the energy consumption is up to $50 \%$ without heat irradiance.

\section{Conflict of Interests}

The authors declare that there is no conflict of interests regarding the publication of this paper.

\section{Acknowledgment}

The authors would like to thank the Ministry of Science and Technology of Taiwan for financially supporting this research through Grants nos. 103-2221-E-327-039-, 103-2623E-110-003-ET, 102-2622-E-110-008-CC2, and 102-2622-E-110006-CC3.

\section{References}

[1] C. A. Tran, C.-F. Chu, C.-C. Cheng et al., "High brightness $\mathrm{GaN}$ vertical light emitting diodes on metal alloyed substrate for general lighting application," Journal of Crystal Growth, vol. 298, pp. 722-724, 2007.

[2] K. R. Hardy, M. S. Olsson, J. R. Sanderson et al., "High brightness light emitting diodes for ocean applications," in Proceedings of the Oceans MTS/IEEE Conference, Vancouver, Canada, October 2007.

[3] W. H. Dorman, "Lighting device for dental and surgical procedures," U.S. Patent 3511983, 1970.

[4] W. H. Dorman, “Reflector," U.S. PATENT 4149227, 1979.

[5] S. A. Michael and F. H. Holt, "Surgical light with conical reflector," U.S. Patent no. 5,913,599, 1999.

[6] H. Ries and J. Muschaweck, "Tailored freeform optical surfaces," Journal of the Optical Society of America A, vol. 19, no. 3, pp. 590595, 2002.

[7] F. Fournier, Freeform reflector design with extended sources [M.S. thesis], 2010.

[8] C. Canavesi, W. J. Cassarly, and J. P. Rolland, "Direct calculation algorithm for two-dimensional reflector design," Optics Letters, vol. 37, no. 18, pp. 3852-3854, 2012.

[9] J. Chen, Q. Zhu, Q. Chen, Q. Luo, X. Zhu, and B. Yu, "Reflector design of light emitting diode for uniform illuminance," in Proceedings of the International Symposium on Photonics and Optoelectronics (SOPO '12), pp. 1-4, May 2012.

[10] H. H. Frazier and A. M. Smith, "Surgical light with conical reflector," STERIS Corporation, US Patent US5913599, 1999.

[11] U. Gampe and S. Greif, "Reflector for a radiating luminous source and use of the same," US6080464A1, 2000.

[12] W. Harry, "Reflector for dental and surgical operating room lighting fixtures," U.S. PATENT 494257, 1990.

[13] Medical electrical equipment particular requirements for the safety of surgical luminaries and luminaries for diagnosis in accordance with IEC-60601-2-41.

[14] “Prp/E, WILDFIRE 5.0,” Needhamm, Mass, USA, 2010.

[15] C. T. Pan, Y. C. Chen, P. H. Lin, C. C. Hsieh, F. T. Hsu, and J. C. Huang, "Lens of controllable optical field with thin film metallic glasses for UV-LEDs," Optics Express, vol. 22, no. 12, pp. 1441114424, 2014.

[16] Y. C. Chen, C. T. Pan, C. C. Hsieh, C. Y. Su, H. C. Wu, and W. C. Li, "Fabrication of light extraction efficiency of organic light-emitting diodes with $3 \mathrm{D}$ aspherical microlens by using dry etching process," Journal of Nanomaterials, vol. 2013, Article ID 797534, 6 pages, 2013. 


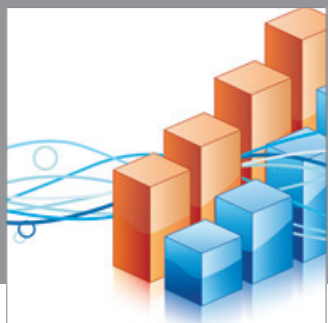

Advances in

Operations Research

mansans

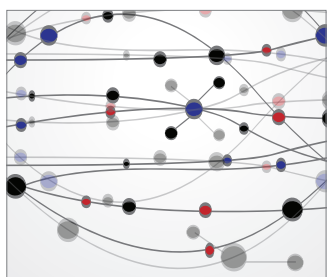

The Scientific World Journal
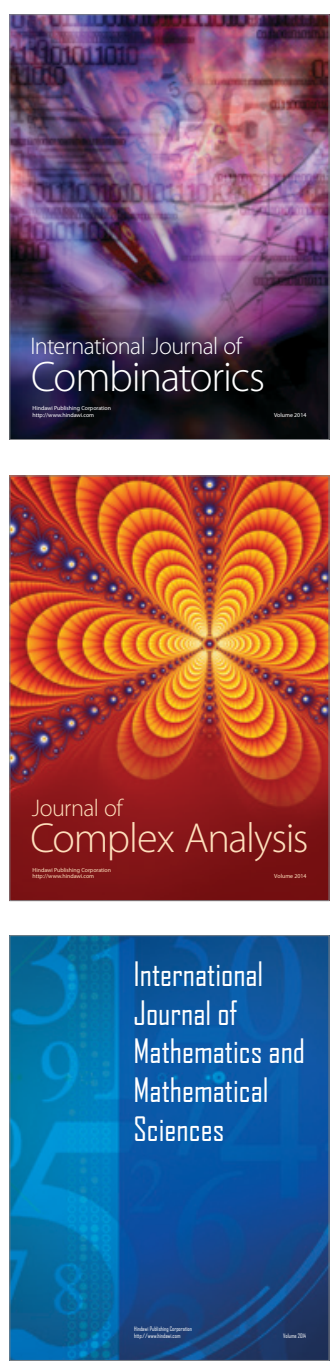
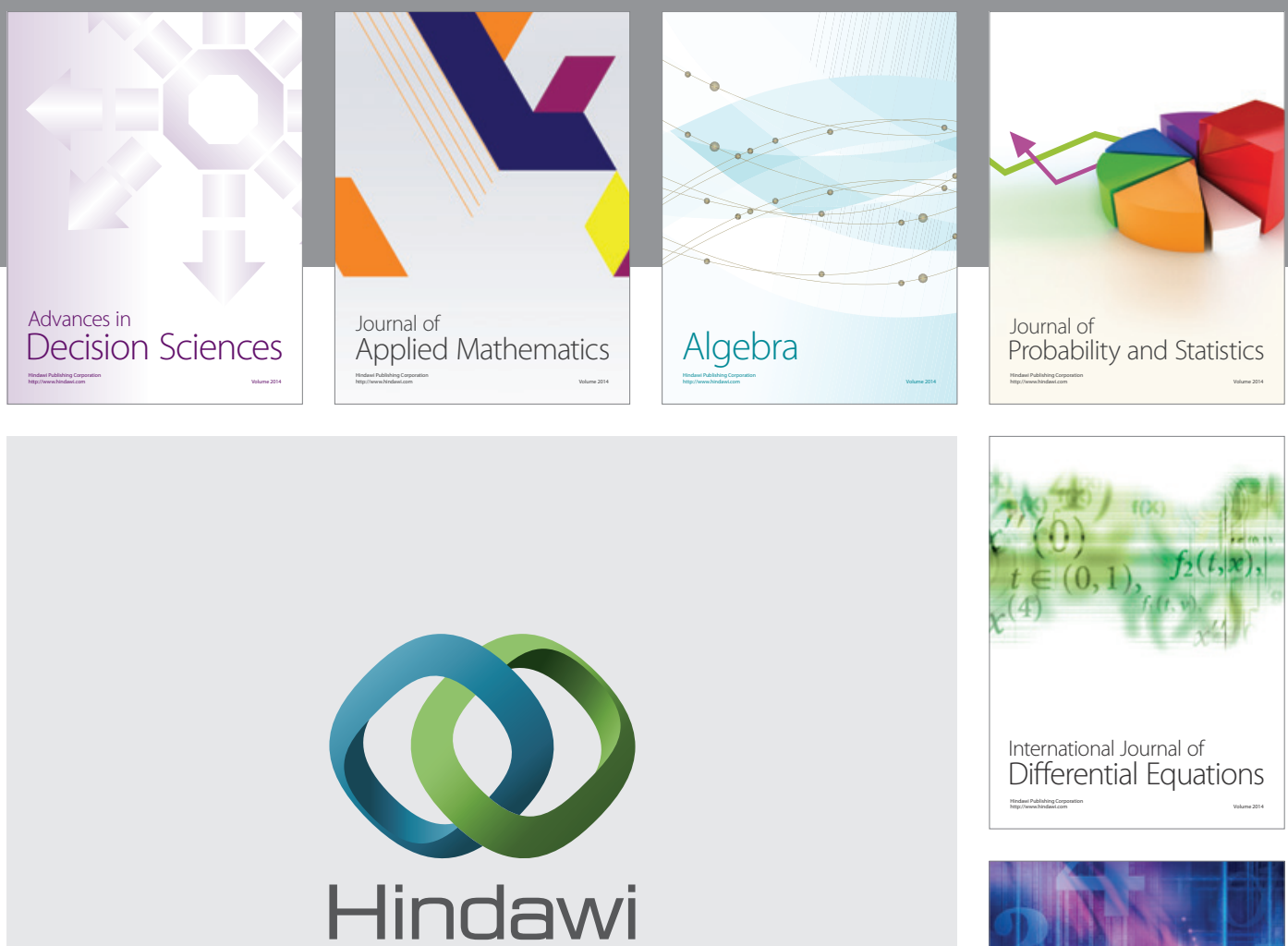

Submit your manuscripts at http://www.hindawi.com
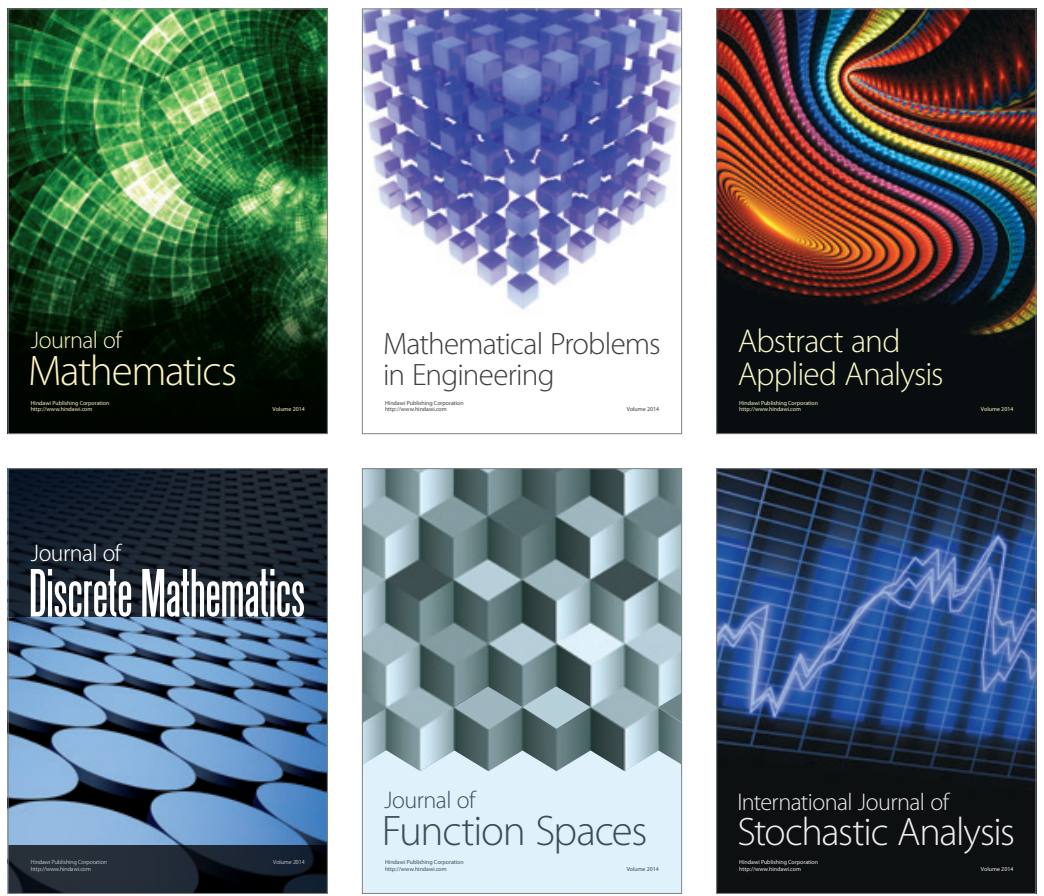

Journal of

Function Spaces

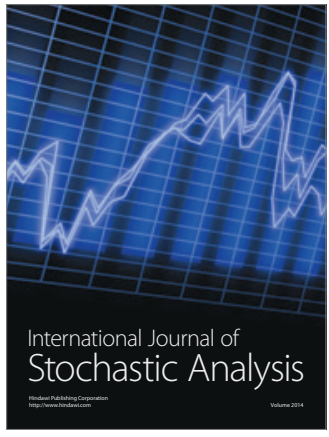

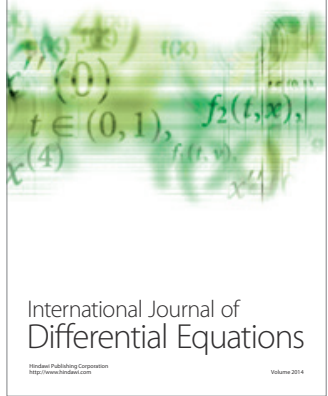
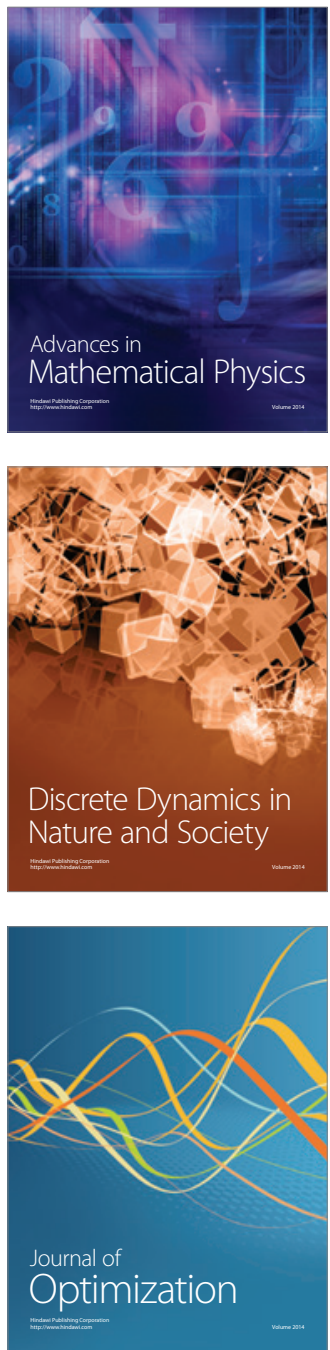